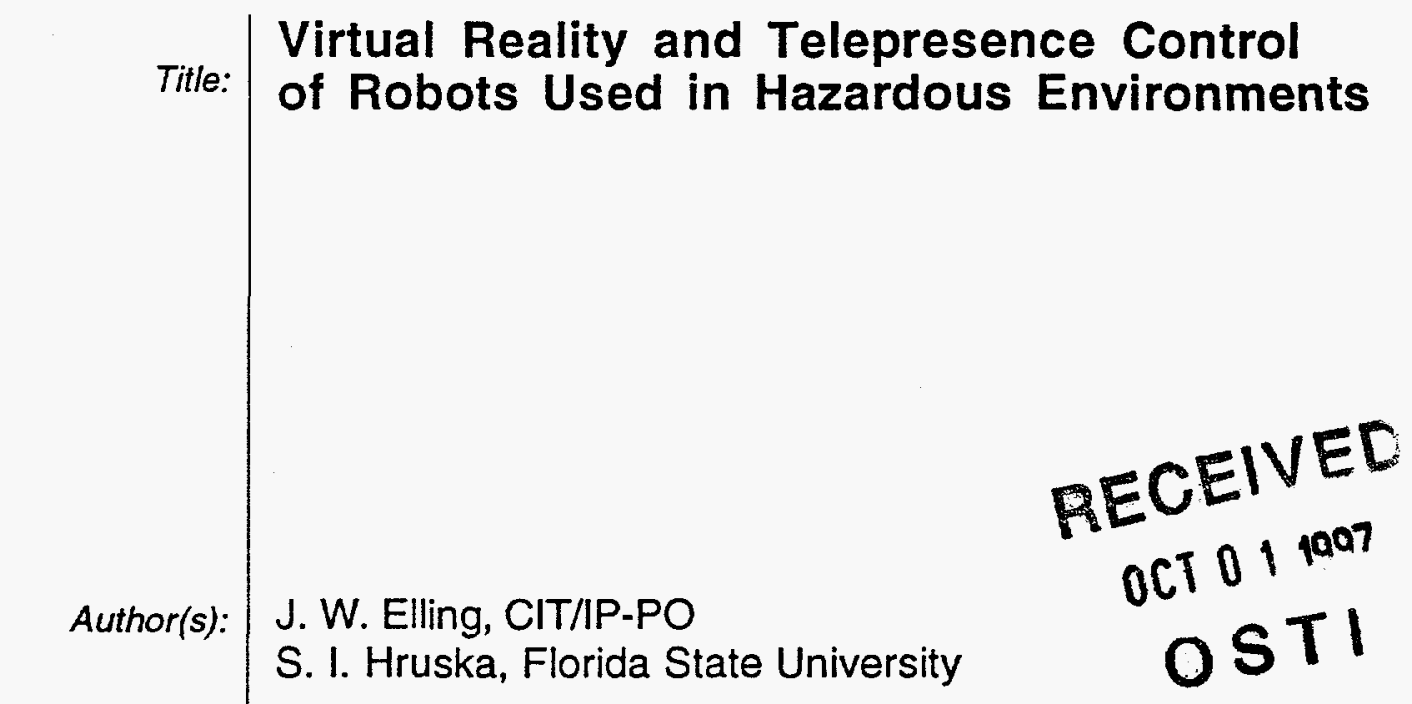

Submitted to: DOE Office of Scientific and Technical Information

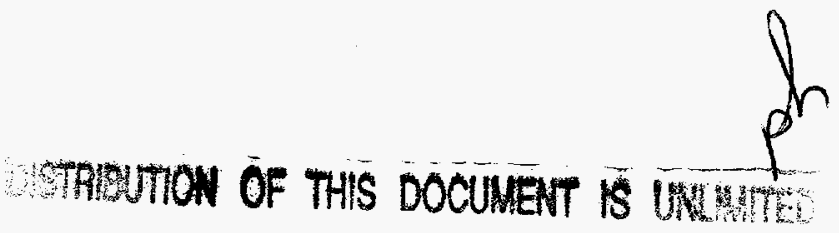

Los Alamos National Laboratory, an affirmative actionequal opportunity employer, is operated by the University of Califomia for the U.S. Department of Energy under contract W-7405-ENG-36. By acceptance of this article, the publisher recognizes that the U.S. Government retains a nonexclusive, royaltyfree license to publish or reproduce the published form of this contribution, or to allow others to do so, for U.S. Government purposes. Los Alamos National Laboratory requests that the publisher identity this article as work performed under the auspices of the U.S. Department of Energy. Los Alamos National Laboratory strongly supports academic freedom and a researcher's right to publish; as an institution. however, the Laboratory does not endorse the viewpoint of a publication or guarantee its technical correctness. 


\section{DISCLAMIER}

Portions of this document may be illegible in electronic image products. Images are produced from the best available original document. 


\section{DISCLAIMER}

This report was prepared as an account of work sponsored by an agency of the United States Government. Neither the United States Government nor any agency thereof, nor any of their employees, make any warranty, express or implied, or assumes any legal liability or responsibility for the accuracy, completeness, or usefulness of any information, apparatus, product, or process disclosed, or represents that its use would not infringe privately owned rights. Reference herein to any specific commercial product, process, or service by trade name, trademark, manufacturer, or otherwise does not necessarily constitute or imply its endorsement, recommendation, or favoring by the United States Government or any agency thereof. The views and opinions of authors expressed herein do not necessarily state or reflect those of the United States Government or any agency thereof. 


\title{
Virtual Reality and Telepresence Control of Robots Used in Hazardous Environments
}

\author{
John W. Elling* \\ Los Alamos National Laboratory \\ Susan I. Hruska \\ Florida State University
}

\begin{abstract}
This is the final report of a two-year, Laboratory Directed Research and Development (LDRD) project at the Los Alamos National Laboratory (LANL). A method was developed to incorporate learning and adaptive capabilities into traditional rule-based descriptions of environments. The learning and adapting capacity is critical to robot programming in highly unstructured and variable environments such as the operation of robots in gloveboxes. The technology is based on a hybrid expert system technology, called expert networks, and was developed with the associated knowledge capture techniques.
\end{abstract}

\section{Background and Research Objectives}

The Automated Retirement and Integrated Extraction System (ARIES) project at Los Alamos National Laboratory (LANL) is currently developing solutions to the problem of recovering plutonium assemblies from decommissioned weapons. The current process involves enclosing the hazardous materials in a glove box, presenting a situation in which the technicians attempt to manipulate objects through bulky, restrictive rubber gloves while exposed to various levels of radiation. The high levels of radiation that are possible and the large quantity of material that will have to be handled make automation of the tasks necessary.

The use of robots and telepresence in combination with dexterous manipulators in a glovebox would allow the operator to perform his task while removed from the hazards. The difficulty of using robots in an unstructured yet constrained environment, such as a glovebox in which many different procedures can be performed, is that the robot must be programmed to accomplish each task. Traditional robot programming is time consuming, and the results are highly specific to a single task. The intent of this research was to develop and test an alternative control technology that may have applicability to robot programming.

\footnotetext{
* Principal Investigator, E-mail: elling @lanl.gov
} 


\section{Logical Programming}

Rule based, algorithmic programs contain a set of logical rules related to the problem domain and represented in forms such as "if $x$ then $y . "$ The programs are typically created by the robot operators for each process. Algorithmic programs have the advantage that the if/then representation of the relationship between inputs and outputs is a natural way to capture the knowledge. Another advantage of algorithmic systems is the easily understood explanation of the outputs in terms of the inputs and path of the if/then structures.

One disadvantage of traditional algorithmic programming is the extreme brittleness of the systems. Once an acceptable program is constructed, changes to any part of the system are problematic. For example, if an additional or modified procedure is incorporated, the interactions with all the other parts of the program must be evaluated in order to avoid the introduction of contradictions and errors. The brittleness also results in a rapid performance degradation if the robot environment modeled by the program changes in any way. For example, if the workspace layout shifts, the program is no longer valid even if the high level procedure being performed is the same.

\section{Artificial Neural Network Control}

Artificial Neural Networks (ANNs) are another traditional control technique. An ANN operates by presenting a set of input signals to the input units, allowing the processing units to propagate these signals throughout the network and come to a steady state. The final results are read at the output units of the network. Learning is achieved by training the connection weights based on a series of known examples such that the ANN learns to recognize patterns in the training inputs.

Artificial neural nets have the advantage of greatly simplifying the program development process since the relationship between inputs and outputs can be learned rather than specified. Given the appropriate ANN architecture, any problem fitting the pattern recognition paradigm can be learned. Artificial neural nets have an additional advantage that, given a well trained network, degraded or noisy inputs usually lead to a graceful degradation of the performance of the network. The result is that the system is less brittle than traditional algorithmic programs. When performance degrades to an unacceptable level, often the network can be quickly retrained.

One primary drawback to the neural network approach is the lack of an accessible explanation of the outputs. The learned relationship between inputs and any output is difficult to discern. Another disadvantage of developing an ANN solution is the need for an extensive training set that may be difficult and/or expensive to obtain. 


\section{Expert Network Control}

Expert network techniques provide a mapping from a rule-based system to a type of artificial neural network and back. ${ }^{1}$ The translated rule-base expert system is called an expert network due to the creation of specialized nodes not common to traditional neural networks. This hybrid approach makes use of traditional algorithmic programming structures while providing a mechanism for data-driven connectionist training. This LDRD project investigated expert networks that may be appropriate for robot control.

\section{Importance to LANL's Science and Technology Base and National R\&D Needs}

The ARIES project at Los Alamos is currently developing solutions to the problem of recovering plutonium assemblies from decommissioned weapons. The current process involves enclosing the hazardous materials in a glove box, presenting a situation in which the technicians attempt to manipulate objects through bulky, restrictive rubber gloves while exposed to various levels of radiation. The high levels of radiation that are possible and the large quantity of material that will have to be handled make automation of the tasks necessary. However traditional robotic automation in a research or variable environment is disadvantaged by the high need for constant robot programming in order to accomplish different tasks. This research explores hybrid programming techniques that may have advantages in robot control.

\section{Scientific Approach and Accomplishments}

Expert network techniques provide a mapping from a rule-based system to a type of artificial neural network and back. ${ }^{1}$ The translated rule-based expert system is called an expert network due to the creation of specialized nodes not common to traditional neural networks. This hybrid approach makes use of traditional algorithmic programming structures while providing a mechanism for data-driven connectionist training.

The translation of algorithmic programs from a rule base to a neural network involves mapping the inputs and outputs onto network nodes. The if/then rule is represented by connections between the nodes, and the rule certainty is represented by the weight of the connection. Translating the conditional statement "if $x$ then $y(\mathrm{cf})$," where $x$ and $y$ are assertions and $\mathrm{cf}$ is a certainty factor, is shown in Figure 1. Assertions $\mathrm{x}$ and $\mathrm{y}$ can be system inputs or outputs or intermediate values in a programmed procedure. 
Logical structures with multiple assertions and logical operators are translated into multiple nodes in the network. Regular nodes represent simple assertions, and operation nodes represent logical conjunctions of assertions or complex assertions. For example the network representation of the rule "if a or b and c then $\mathrm{d}(\mathrm{cf})$ " is shown in Figure 2. The unlabeled connections between nodes in Figure 2 implicitly carry a weight of 1.0.

Expert networks preserve the easily understood procedural representation and explanation capability while providing the learning capability of network systems. Once in network format, the program can be trained with connectionist-style learning which optimizes the node connection weights as well as parameters of internal node functions. The expert network representation also has the robustness that results from the ability to periodically retrain the system to adapt to changes in the robot environment.

\section{Our System}

We implemented an automated system diagnostic and execution program with a four-layer expert network consisting of Input, Filter, Combination, and Output nodes. Inference chains are constructed for each output by connecting all symptoms related to the fault through appropriate Filter nodes based on the semantic qualifier to a Combination node. This is in turn connected to the Output node, as shown in Figure 3. Figure 3 illustrates the sub-network architecture for each system output.

In the sub-network, for each output there is one Input-node-to-Filter-node pair for each connection in the knowledge table. The inferencing mechanism for this expert network architecture first propagates the inputs to their connected Filter nodes in all the subnetworks in parallel. In the Filter nodes, the inputs are compared with a threshold. Values that exceed the threshold represent positive evidence for that output. Values that do not exceed the threshold are subtracted from 1.0, and the result is negative evidence for the output.

The reinforcement factor in the Filter node is used to positively reinforce input values that exceed the threshold. All input values exceeding the threshold are multiplied by the reinforcement factor. In effect, this passes the full-strength input value to the combination node. Penalty factors in the Filter nodes serve to convert small positive input values, that are below the threshold, into negative evidence. The weight of the outgoing connection from the Filter node is also trainable.

The filtered and weighted inputs become evidence for an output that is combined in the Combination nodes. Combination nodes synthesize positive and negative evidence into a conclusion of the sub-network fault with an EMYCIN-like formula. ${ }^{2}$

The training algorithms can modify the values of the reinforcement factor, the penalty factor, and the outgoing weight for each Filter node individually in the network. 
The primary goal of the training is to increase the predictive capability of the system by optimizing the trainable parameters. Training optimizes the inferencing process for each input individually in each subnetwork.

\section{Initial Results}

The system was implemented and tested on an instrument fault diagnosis problem. After training, the network shows improved diagnostic ability. The untrained expert network implementation was able to correctly diagnose the induced fault in 299 of the 718 samples, or $41.6 \%$. After training the Filter-node-to-Combination-node connection weights, the network was able to correctly diagnose 556 of the training set correctly, increasing the accuracy to $77.4 \%$.

\section{Multiple Paths of Reasoning}

In some cases there may be more than one logical pathway to an output. The appearance of a subset of inputs would then be adequate to produce that output. When this is the case, there is said to be multiple paths leading to this output. Multiple paths are created in an expert network by adding additional Combination nodes connected to the Output node in the subnetwork. Figure 4 represents the two parallel paths in the subnetwork. All combination nodes related to the common fault are linked to the same output node. ${ }^{3}$

It is sometimes difficult to determine the number of paths appropriate for each output. For this reason multiple paths were determined directly from training data using a self-organizing clustering technique, ART2. ART2 is an implementation of Adaptive Resonance Theory and is a self-organizing network capable of clustering data using an unsupervised learning technique. 4,5 The advantage of this technique is that it does not require a-priori specification of the number of clusters in the data.

If two or more definitive clusters are created from the data for a training example, each cluster is suggested as a multiple reasoning path for that example. A definitive cluster is a cluster which contains a significant fraction of the total number of samples for that example. Details of the clustering study are available in Ref. 3 .

\section{Results with Multiple Path Knowledge}

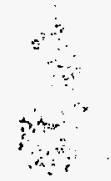

By incorporating knowledge for multiple paths of reasoning into the system and the expert network, the performance improves. The original expert network was able to correctly diagnose 296 of the 667 training data files, or $44.4 \%$. After training on these data files, this expert network was able to correctly diagnose 591 of the training data, or $88.6 \%$. 
The expert network built with parallel paths correctly predicts 475 of the 667 files before training, or $71.2 \%$. After training the expert network with parallel paths correctly identifies 638 of the 667 training files, or $95.7 \%$.

\section{References}

1. D. C. Kuncicky, S. I. Hruska, R. C. Lacher, "Hybrid systems: The equivalence of rulebased expert systems and artificial neural network inference," International Joumal of Expert Systems, vol. 4, no. 3, pp. 281-297, 1992.

2. Alan P. Levis, Robert G. Timpany, W. E. Austad, J. W. Elling, J. J. Ferguson, D. A. Klotter, S. I. Hruska, "Application of Knowledge-based Network Processing to Automated Gas Chromatography Data Interpretation," SPIE Vol. 2492, Application and Science of Artificial Neural Networks, pp. 294-302, (1995).

3. Kristin L. Adair, "Data Driven Optimization of Expert Networks," Master's thesis, Florida State University, Tallahassese, Florida, Fall 1996.

4. Gail A. Carpenter and Stephen Grossberg, "The Art of Adaptive Pattern Recognition by a Self-Organizing Neural Network," IEEE Computer, vol. 21, no. 3, pp. 77-88, 1988.

5. Gail A. Carpenter and Stephen Grossberg, Pattern Recognition by Self-Organizing Neural Networks, Chapter ART2: "Self-Organization of Stable Category Recognition Codes for Analog Input Patterns," pp. 397-423, MIT Press, Cambridge, Massachusetts, 1991. 


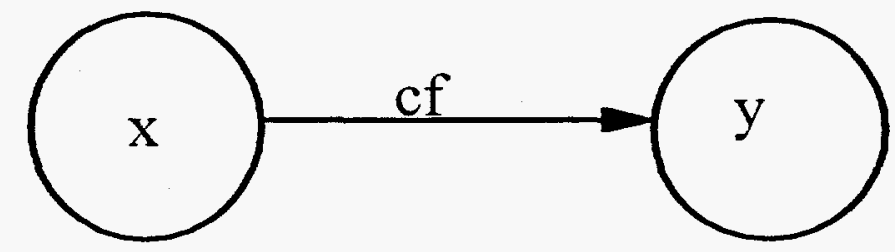

Figure 1. Expert network representation of an "if $x$ then $y$ (cf)" rule.

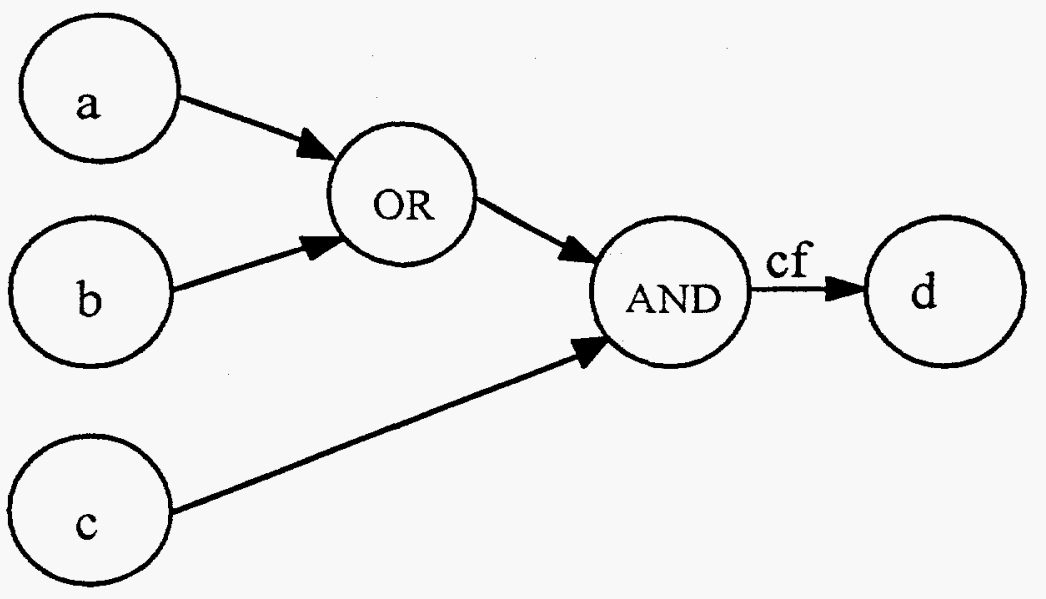

Figure 2. Expert network representation of an "if $a$ and $b$ and not $c$ then $d$ (cf)" function. 


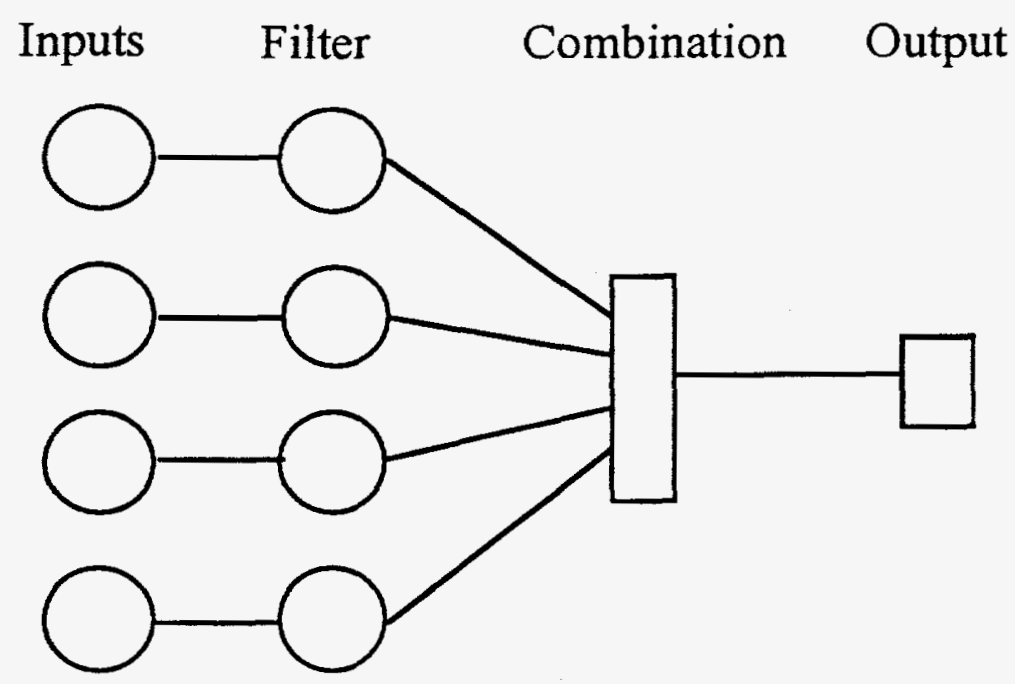

Figure 3. The expert network architecture.

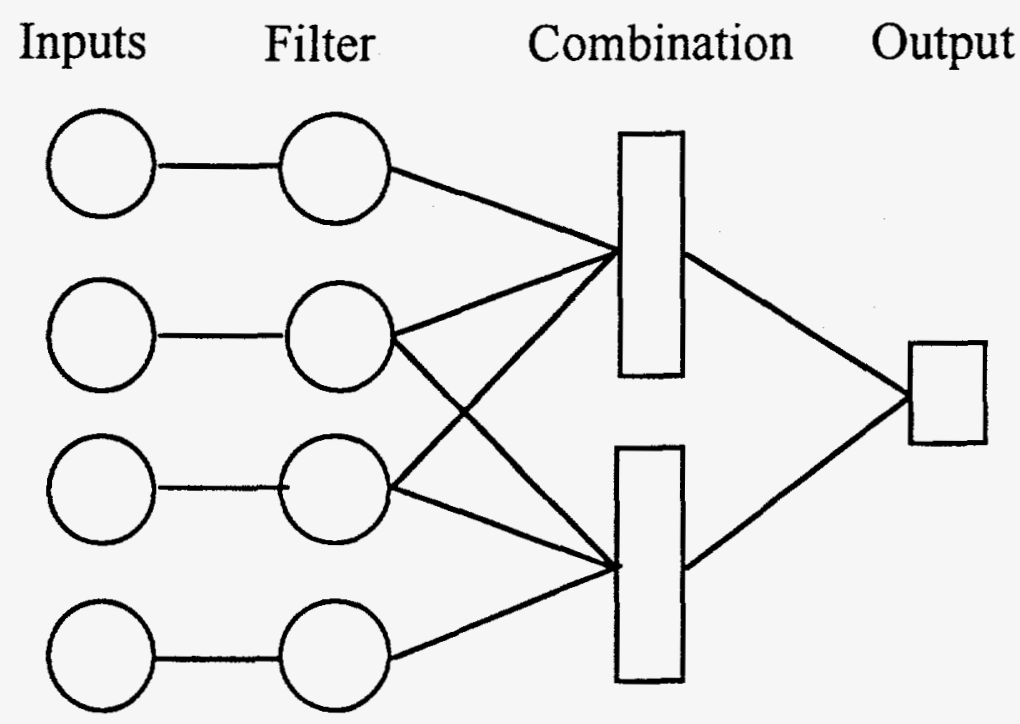

Figure 4. Network architecture for two paths of reasoning. 